\title{
Cost-effectiveness of surgical interventions for the management of osteoarthritis: a systematic review of the literature
}

\author{
Hanin Kamaruzaman ${ }^{1}$, Philip Kinghorn ${ }^{2}$ and Raymond Oppong ${ }^{2^{*}}$ (D)
}

\begin{abstract}
Background: The primary purpose of this study is to assess the existing evidence on the cost-effectiveness of surgical interventions for the management of knee and hip osteoarthritis by systematically reviewing published economic evaluation studies.

Methods: A systematic review was conducted for the period 2004 to 2016. Electronic databases were searched to identify both trial and model based economic evaluation studies that evaluated surgical interventions for knee and hip osteoarthritis.

Results: A total of 23 studies met the inclusion criteria and an assessment of these studies showed that total knee arthroplasty (TKA), and total hip arthroplasty (THA) showed evidence of cost-effectiveness and improvement in quality of life of the patients when compared to non-operative and non-surgical procedures. On the other hand, even though delaying TKA and THA may lead to some cost savings in the short-run, the results from the study showed that this was not a cost-effective option.

Conclusions: TKA and THA are cost-effective and should be recommended for the management of patients with end stage/severe knee and hip OA. However, there needs to be additional studies to assess the cost-effectiveness of other surgical interventions in order for definite conclusions to be reached.
\end{abstract}

Keywords: Osteoarthritis, Cost-effectiveness, Costs, Review

\section{Background}

Osteoarthritis (OA) is the most common form of joint disease, and results from a progressive degenerative change in the joint structure. OA is associated with any joint in the body but the most commonly affected are the hip and the knee [1]. It has been estimated that about 251 million people suffer from knee OA worldwide [2]. The prevalence of OA increases with age, and with the constant rise in the global ageing population $[3,4]$, the economic burden of the disease is also likely to rise.

Osteoarthritis places a strain on scarce resources. For example, in a recent study, the total annual direct cost of osteoarthritis in the US was estimated to be double

\footnotetext{
* Correspondence: r.a.oppong@bham.ac.uk

${ }^{2}$ Health Economics Unit, Institute of Applied Health Research, University of

Birmingham, Birmingham, UK

Full list of author information is available at the end of the article
}

that of similar patients who did not have osteoarthritis [5]. In the UK, the total health care cost of osteoarthritis is estimated at over $£ 1$ billion (2010 prices) [6]. Based upon national survey data, Kortlarz et al. estimate the increased insurer expenditure for women in the US with osteoarthritis to be $\$ 4,833$ [7]. For men with osteoarthritis, the additional insurer cost was estimated as $\$ 4,036$ [7]. There is therefore a need for cost-effective approaches for the management of OA.

Treatment and management of OA involves a multidisciplinary approach and various management options include patient education and self-management, nonpharmacological treatments and pharmacological treatments. In the recently updated clinical guidelines for management of OA, issued by the National Institute for Health and Care Excellence (NICE), comprehensive and integrated care which involves healthcare professionals, patients and carers are among the key recommendations 
intended to ensure the maximum benefit for patients [8]. Apart from these pharmacological and nonpharmacological interventions, there are also surgical interventions which are more expensive and are normally limited to those patients who do not respond to other forms of treatment. Due to the increase in the number of older people, there has been an increase in the demand for surgical interventions such as joint arthroplasty which has caused a rise in the costs associated with OA [9-11].

Systematic reviews have assessed the cost-effectiveness of pharmacological and non-pharmacological treatments for OA [12-14]. However to the best of our knowledge, a systematic review of economic evaluations assessing surgical interventions for the management of OA has never previously been published or reported. This study is therefore aimed at reviewing published economic evaluations aimed at assessing and summarizing evidence on the cost-effectiveness of surgical interventions for the management of OA. The focus is on knee and hip OA since they are the most common forms of OA.

\section{Methods}

The search and identification of papers followed a twostage process. The initial search included economic evaluations associated with any clinical trials or cohort studies that assessed surgical, non-pharmacological and pharmacological interventions for all types of OA. This was necessary to meet the needs of the wider research project. The search strategy outlined below reflects this initial, broad scope.

\section{Search strategy}

Electronic databases (Ovid MEDLINE(R) In-Process \& Other Non-Indexed Citations and Ovid MEDLINE(R), Ovid EMBASE Classic and EMBASE, EBM ReviewsCochrane Database of Systematic Review, EBM ReviewsNHS Economic Evaluation Database, PubMed, INAHTA database and HTA websites) were searched for economic evaluations associated with any clinical trials or cohortbased trials that assessed all types of treatment modalities (pharmacological, non-pharmacological, surgical) for all types of OA.

Additional literature which was considered as potentially relevant was identified from the bibliographies of the retrieved articles and search engines such as Google Scholar. Any studies with limited access were retrieved by emailing the author(s) and no language restrictions were imposed. The searches were conducted between 2004 and 2016. This timeframe was chosen for pragmatic reasons and in order to capture the most recent studies. The detailed search terms that were used, has been outlined in Additional file 1.

\section{Selection of studies}

All titles and abstracts of retrieved articles were screened and any duplicates were excluded from the initial list. Each article was then checked for suitability in terms of their potential in assessing the cost-effectiveness and economic analysis of various treatment modalities for management of OA. Full text articles of these selected titles were retrieved and reviewed for further selection in accordance to pre-specified inclusion and exclusion criteria. The selection of articles was carried out independently by two reviewers $[\mathrm{HK}]$ and [RO]. Results from each reviewer were compared and any differences were resolved through consultation among reviewers.

It was from this broad pool of papers that the following, more specific, inclusion and exclusion criteria were applied, in order to identify studies falling within the scope of this paper.

\section{Inclusion criteria}

The inclusion criteria for this systematic review were divided into 5 main components as follows:

i. Population: Patients with knee or hip osteoarthritis

ii. Intervention: Surgical interventions (total knee arthroplasty, total hip arthroplasty etc).

iii. Comparators: Any comparators (no interventions, usual care, and other surgical modalities)

iv. Outcomes: Any outcomes for economic evaluations (cost effectiveness, incremental cost-effectiveness ratio or ICER)

v. Study design: Cost consequence analysis, costbenefit analysis, cost-effectiveness analysis, costutility analysis both trial-based studies and modelbased economic analysis

\section{Exclusion criteria}

The exclusion criteria for this review were as follows:

i. Study designs that do not consider knee or hip OA or study designs that combine OA patients with other types of arthritis (rheumatoid arthritis, lupus arthritis, ankylosing spondylitis)

ii. Partial or non-economic evaluation studies

iii. Ongoing studies and protocol

iv. Systematic reviews, narrative review, commentaries and letters

v. Duplicated publications

\section{Data extraction and methods of analysis and synthesis}

The following data was extracted from the selected studies: Study characteristics such as type of economic analysis, population, interventions, comparators, perspective, time horizon, and types of modelling used, effectiveness and cost measures, main results, ICER, base-case outcomes 
and sensitivity analyses being used. A summary of the studies relating to surgical interventions for the treatment of hip and knee OA is presented in Table 1 .

\section{Assessment of the quality of studies}

Assessment of the quality of included studies was performed by using the Consolidated Health Economic Evaluation Reporting Standards or CHEERS [15] (Additional file 1) and Philips criteria checklist [16] (Additional file 1) for model based studies. All included studies were also quantitatively assessed using the Quality of Health Economic Studies (QHES) instrument (Additional file 1) which was designed to evaluate the appropriateness of the methodology, the validity and transparency of the study results and the comprehensiveness of reporting the study itself [17].

\section{Results}

\section{Search results}

The initial (broad) search yielded 303 potentially relevant articles and after reviewing the abstracts and applying the inclusion criteria, 121 studies were initially included for full text review. After applying exclusion criteria set out above, a total of 98 articles were excluded, mainly because they were duplicates (6 studies), were not concerned with assessing surgical interventions (38 studies), not limited to knee and hip OA patients (19 studies), not full economic evaluations (22 studies), protocols (3 studies) and systematic reviews (10 studies). A total of 23 studies were included in the final sample for this paper (Fig. 1).

\section{Summary of selected studies}

All studies included in this review were published between 2004 and 2016 and were conducted across nine different countries (USA, Italy, Canada, Australia, Finland, UK, Singapore, Belgium and Romania). The types of economic evaluations conducted were mainly cost-utility analysis (78\%) and cost-effectiveness analysis (13\%) with one study conducting a cost-benefit analysis [18] and another conducting both a costutility analysis and a cost-effectiveness analysis [19]. Sixteen studies were model based, whilst six were trial based (Table 1). Most studies adopted either a healthcare (nine studies) or a societal perspective (eight studies). Two studies however adopted both a healthcare and a societal perspective $[19,20]$. Of the remaining studies, four did not specifically mention the perspective that was adopted [21-24].

Three studies only incorporated direct medical costs in their analysis even though they stated that they adopted a societal perspective [25-27]. Most studies described the comparators that were used; one study stated that the comparator of choice was a non- operative strategy [28] but did not give any specific details about the non-operative strategy used. For studies that used the QALY as an outcome measure, various health-related quality of life (HR-QoL) instruments such as the SF-36, EQ-5D, SF-6D, 15Dimensional Utility Index (15D), Health Utility Index (HUI) and Health Assessment Questionnaire (HAQ) were used. Two studies [25, 29], however, did not specifically mention the tools that were used to derive the QALY estimates. Apart from the generic HR-QoL instruments, some studies utilised disease-specific health state instruments. For instance, Räsänen et al [28] used Harris Hip Score (HHS) and Knee Society Score (KSS) as complementary disease-specific tools for the $15 \mathrm{D}$ generic instrument used in the study. Other disease-specific tools used were Oxford Hip Score (OHS), Oxford Knee Score (OKS) and Western Ontario and McMaster Universities Osteoarthritis Index (WOMAC). The most widely used tool by healthcare providers in assessing OA patients is WOMAC Osteoarthritis Index which consists of 24 items used to evaluate pain, stiffness and physical functions of OA patients in daily living [30].

\section{Cost-effectiveness of interventions for the management of knee and hip OA}

For surgical modalities for knee OA, the most common intervention was total knee arthroplasty whilst the most common surgical modality for hip OA was total hip arthroplasty.

\section{Cost-effectiveness of Total Knee Arthroplasty (TKA) TKA versus non-surgical/non operative strategies}

Four studies compared TKA to non-operative/nonsurgical strategies $[18,28,31,32]$ and all studies concluded that TKA is a cost-effective intervention. Of these, three studies adopted a societal perspective $[18,31,32]$ whilst the fourth study [28] adopted a healthcare provider perspective. The aim of the first study [18] was to assess the cost-effectiveness of TKA in the younger working population and used a Markov model to assess the cost-effectiveness of TKA over a 30 year period in a hypothetical 50 year old patient with severe OA. The second study also used a Markov model to estimate the lifetime costeffectiveness of TKA in patients with end stage OA of the knee [31]. The remaining two studies [28, 32] assessed the short term cost-effectiveness of TKA using trial-based and cohort studies and did not limit the population included in their respective studies by age or stage of the disease. A summary of results is presented in Table 2 . 


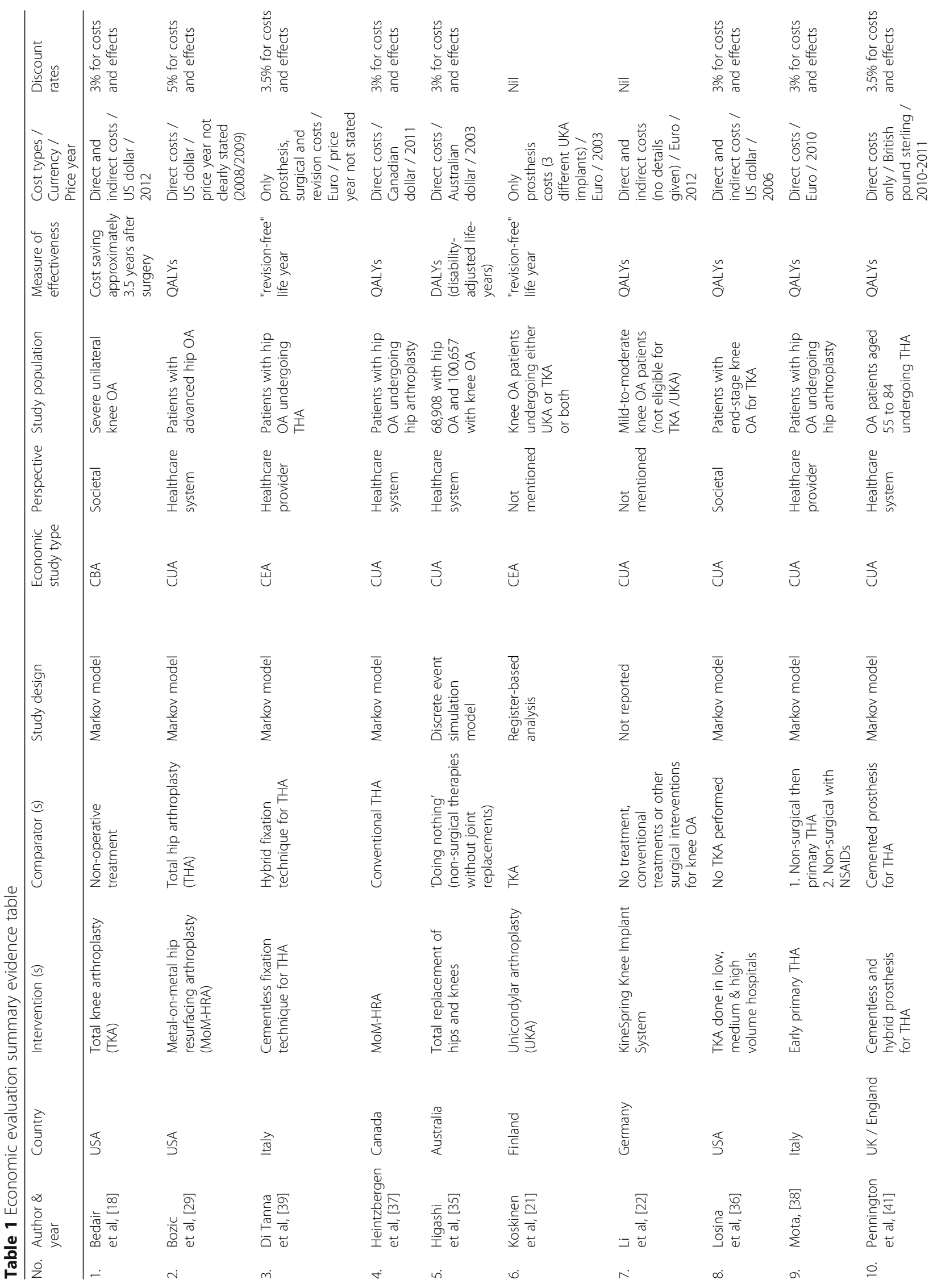


Kamaruzaman et al. BMC Musculoskeletal Disorders (2017) 18:183

Page 5 of 17

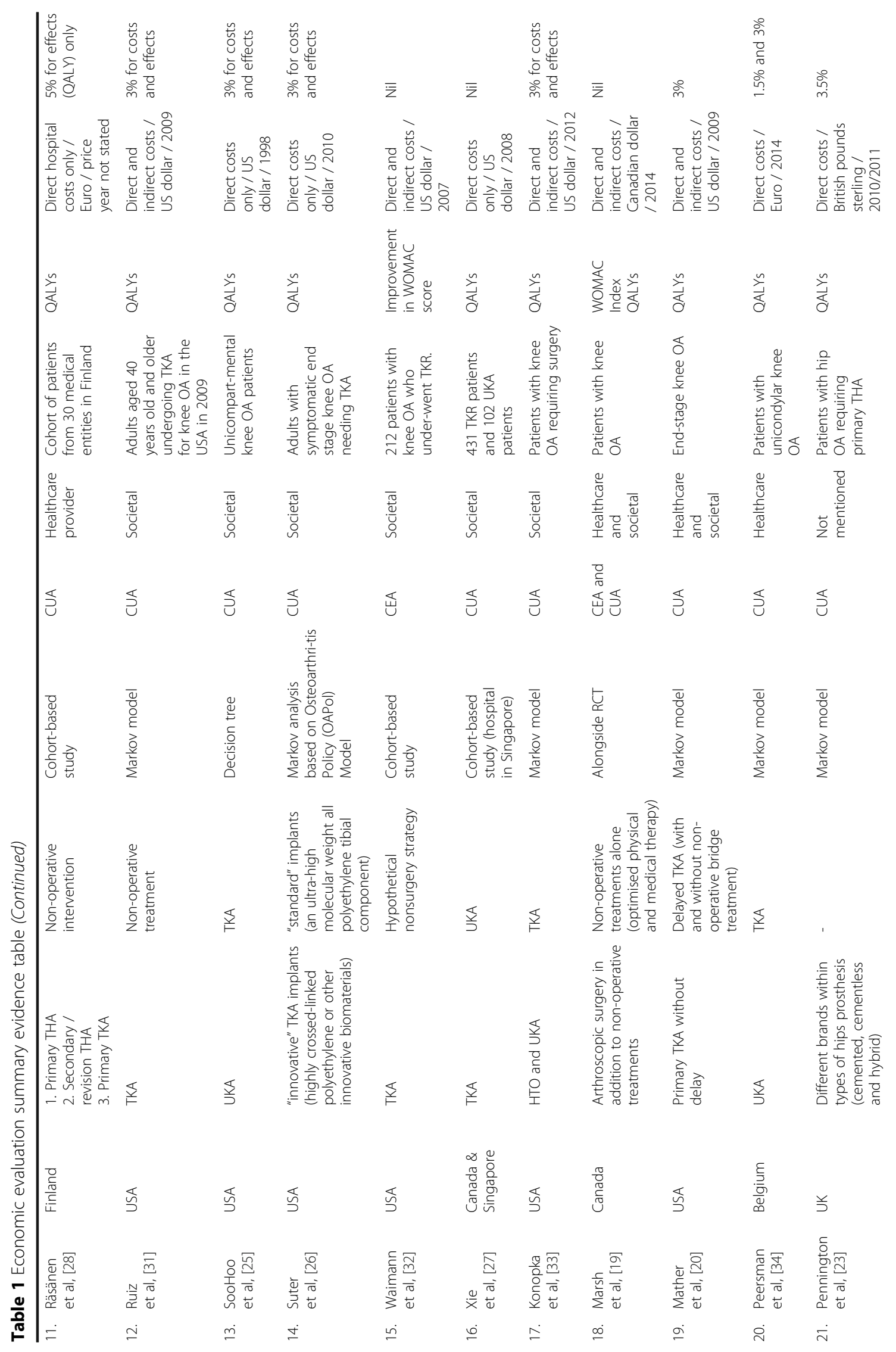




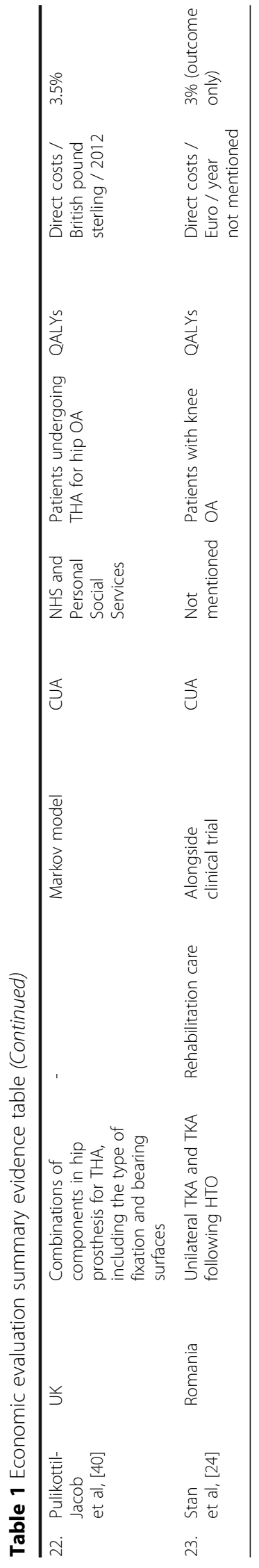




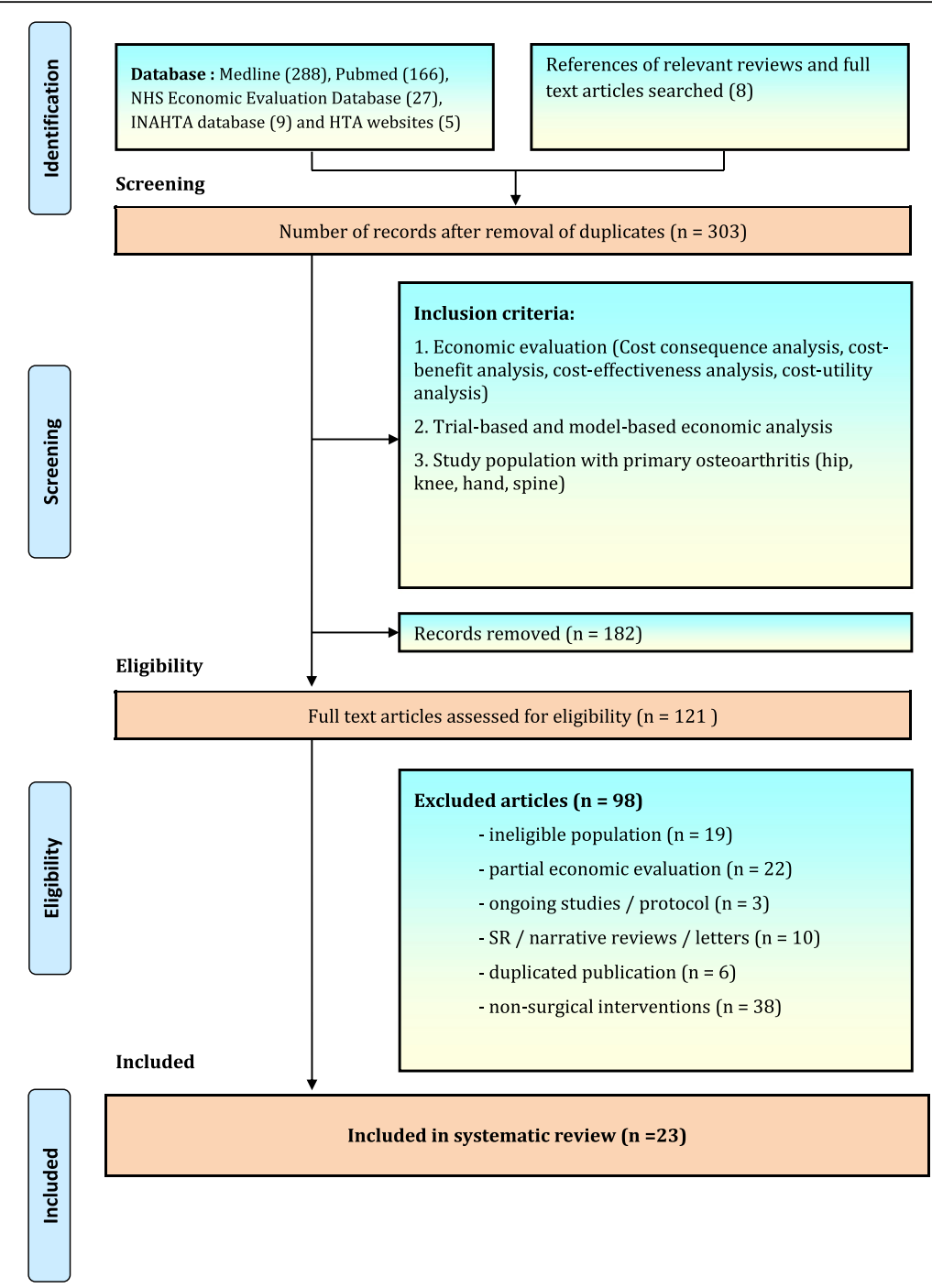

Fig. 1 Flow diagram of the study selection process

\section{TKA versus Unicondylar/Unicompartmental Knee Arthroplasty (UKA)}

Five studies compared TKA with UKA to determine the most cost-effective option [21, 25, 27, 33, 34]. The first study [27] looked at cost-effectiveness over 2 years using a prospective observational study and found that TKA was associated with both a higher cost and a QALY gain, but could not be $95 \%$ confident that TKA is costeffective due to the short length of their study. The second study [21] found that TKA was associated with higher costs and longer hospital stays in the short run whilst UKA was associated with significantly poorer long term survival rates and higher possibility for revision surgery or secondary TKA. The study noted that the cost saved by lower implant prices and shorter hospital stays may not be able to cover the cost of extra revision surgeries that are needed in the future [21]. Peersman et al. [34] used a Markov model to assess cost-effectiveness in four different age groups $(<55,55-65,65-75$ and $75+)$ and found that in all age groups UKA was associated with cost reductions and health gains. The conclusion from this study was that TKA was not cost-effective when compared to UKA. However, this study did not adopt a societal perspective. The study by Soohoo et al. [25] used a decision model to assess cost-effectiveness in patients with end stage unicompartmental knee OA and found UKA to be cost-effective compared to TKA if the durability and function of UKA are assumed to be the same as TKA. The final study [33] compared TKA with UKA and High Tibial Osteotomy (HTO) in younger patients (50-60 years) with unicompartmental OA using a Markov model and from a societal perspective. The study concluded that TKA had just a $31 \%$ chance of being cost-effective compared with 
Table 2 Summary of main results

\begin{tabular}{|c|c|c|c|}
\hline No. & Author \& Year & Intervention(s) evaluated & Key result(s) \\
\hline 1. & Higashi et al, [35] & $\begin{array}{l}\text { Total replacement of hip and } \\
\text { knee }\end{array}$ & $\begin{array}{l}\text { Both hip and knee replacements } \\
\text { were cost-effective compared to } \\
\text { 'doing nothing' at the pre- } \\
\text { defined threshold level of AUD } \\
50,000 \text { per DALY. } \\
\text { - THR : AUD } 7100 \text { to } 15000 \text { per } \\
\text { DALY with different time cost \& } \\
\text { cost offset } \\
\text { - TKR : AUD } 15000 \text { to } 26000 \text { per } \\
\text { DALY with different time cost \& } \\
\text { cost offset }\end{array}$ \\
\hline
\end{tabular}

2. Bedair et al, [18] Total knee arthroplasty (TKA)

3. Losina et al, [36]

4. Ruiz et al, [31]

Total knee arthroplasty (TKA)

5. Waimann et al, [32] Total knee arthroplasty (TKA)

5. Waimann et al, [32] Total knee arthroplasty (TKA)

Total knee arthroplasty (TKA) performed in low, medium and high volume hospitals
Treatment with TKA has a higher initial cost, but the cost benefit in favor of TKA approximately

3.5 years after surgery (a difference of US\$69,800 over the same time period when treated with non-operative strategies

- Base-case ICER : US\$18300 /

QALY

- If willingness to pay (WTP) to improve QOL were set at US\$50 000 per QALY, TKA had a 93\% chance of being the preferred choice (ie, TKA had the highest net benefit) compared with no TKA.

- Low-risk patients : 96\% chance that TKA would be preferred to no TKA if WTP US\$50 000 per

QALY

- High-risk patients : 83\% chance that TKA would be preferred to no TKA

- Relative to nonsurgical treatment, the mean lifetime net societal savings per patient resulting from TKA was US\$18,930

- Each TKA increased lifetime direct costs by a mean of US\$20,635, while the societal savings in lower indirect costs from improved functional status averaged US\$39,565.

- Considering only direct costs, the average ICER was US\$5656 per QALY gained for TKA in the entire cohort and US\$12,410 for those of 80 years old and older.

- The ICERs for WOMAC improvement at 6 months were as follows:

1) US\$33,345 to achieve an MCID 2) US\$25,255 per each WOMAC20 improvement, 3) US\$35,274 per each WOMAC50 improvement 4) US\$56,908 per each WOMAC70 improvement - TKA would be a cost-effective intervention if the WTP amount for the minimum clinically significant absolute or relative improvement were US\$50,000.
Conclusion $\quad$ QHES

scores $^{a}$

Both hip and knee replacements are cost-effective interventions to improve the quality of life of people with OA.

The total economic cost to society for treatment of severe knee OA in a relatively young working person is markedly lower with TKA than it is with non-operative treatment.

- TKA appears to be costeffective in the US Medicareaged population, as currently practiced across all risk groups. - Policy decisions should be made on the basis of available local options for TKA.

- However, when a high-volume hospital is available, TKAs performed in a high-volume hospital confer even greater value per dollar spent than TKAs performed in low-volume centres.

Overall, TKA was cost-effective across all age groups, assuming a willingness-to-pay threshold of US\$50,000 per QALY gained taken from societal perspective.

Although there was no established WTP value for WOMAC change, TKA appeared to be a cost-effective intervention for end-stage knee OA at both low and high levels of improvement in the patients' pain and function. 
Table 2 Summary of main results (Continued)

6. Xie et al, [27]

7. Koskinen et al,[21]

8. SooHoo et al, [25]

9. Li et al, 2013 [22]

10. Suter et al, [26]
Total knee arthroplasty (TKA)

Unicondylar knee arthroplasty (UKA)

Unicondylar knee arthroplasty (UKA)

KineSpring Knee Implant System - intermediate treatment between conservative care and joint-altering surgery targeting the treatment gap in knee OA patients.

"innovative" TKA implants
- ICUR was US\$65,245/QALY from the societal perspective.

- The probability of TKA being a cost-effective strategy is less than 0.4 from the societal or patients' perspective if the WTP is US\$50,000/QALY.

- In contrast, the probability that TKR is a cost effective strategy is 0.7 from the government's perspective if the WTP is only US\$10,000/QALY

- The mean cost of one revision from UKA to TKA was $€ 8,660$ including implant, hospital stay, operation, and other direct costs. Thus, the costs saved by lower implant prices and shorter hospital stay for UKA as compared to TKA would not cover the costs of the extra revisions.

- In reference case, UKA has only small gain of QALY (0.02) and minimal increment in costs, from US\$18,995 to US\$19,000

compared to TKA

- Reference case ICER : US\$277

per QALY gained.

- In lower durability / survival of

UKA in terms of function, UKA

becomes less effective and more costly.

- If durability / survival of TKA is longer (range 15 to 20 years),

TKR becomes more cost

effective.

- If TKR durability is 20 years, the ICER for UKA would be $\$ 45,958$ per QALY gained when UKA is assumed to be functional up to 17 years ("below threshold)

- Assuming the durability of 10 years, the cost-utility ratio of each intervention compared to no treatment :

- KineSpring : $€ 3,402 \pm 4,168 /$

QALY,

- Surgical interventions : $€ 4,899 \pm$

1,094/QALY

- Conservative treatments :

$€ 9,996 \pm 13,612 / \mathrm{QALY}$

- Innovative implants offered $\geq 50 \%$ decrease in long-term TKA failure at $\leq 50 \%$ increased cost offered ICERs $<$ US\$100,000 regardless of age or baseline comorbidity.

- Innovative implant provided a $20 \%$ decrease in long-term failure at $50 \%$ increased cost provided ICERs < US\$150,000 per QALY gained only among healthy 50 59-year-olds.

- Increasing short-term failure, consistent with recent device
TKA gained more OALYs at higher costs compared to UKA. However, a long-term prospective study is necessary to determine the cost-effectiveness of TKR and UKA.

At a nationwide level, UKA had significantly poorer long-term survival than TKA. UKA did not even have a theoretical cost benefit over TKA in the study. Based on the results, widespread use of UKA in the treatment of unicompartmental OA of the knee cannot be recommended.

This analysis demonstrates the potential for UKA to be a costeffective alternative to TKA, depending on the cost as well as the durability and function of a UKA.

The KineSpring Knee Implant System for knee OA is a costeffective strategy over other surgical and conservative treatments for patients in Germany.

Innovative implants must decrease actual TKA failure, not just radiographic wear, by 50 $55 \%$ or more over standard implants to be broadly costeffective.
62 
Table 2 Summary of main results (Continued)

11. Mota, [38]

Early primary THA

12. Räsänen et al, [28]

13. Bozic et al, [29]

14. Heintzbergen et al, [37]

15. Di Tanna et al, [39]
1. Primary THA

2. Secondary / revision THA

3. Primary TKA

Metal-on-metal hip resurfacing arthroplasty (MoM-HRA)

Metal-on-metal hip resurfacing arthroplasty (MoM-HRA) failures, reduced cost-

effectiveness in all groups.

- Early THA has cost-effectiveness ratios of $€ 4100$ or below in all cases.

- Among 80-year-olds, early THA is (extended) dominant $=$ ICER of $€ 20,406$.

- Delayed THA is not costeffective at any threshold for base-case scenario.

- At age 65 years, the ICER for

THA over delayed THA was $€ 987$ in men and $€ 466$ in women.

- The cost per QALY gained (ICUR) was lowest in the primary THA group , followed by primary TKA \& revision THA.

- Primary THA : €6710 per QALY

gained

- Primary TKA : €13,995 per QALY

gained

- Revision THA : €52,274 per QALY gained

- Lowest ICER [most costeffective] : men age 55 to 64 (US\$28,614/QALY gain)

- Three groups with ICER below threshold [below US\$50,000/ QALY gained) :

- men age 55 to 64 (as above) - women younger than 55 (US\$47,468/QALY gained)

- men younger than 55 (US\$48,882/ QALY gained)

- Base-case : MoM HRA dominates with -CAD \$583 and mean difference QALY 0.079

- With WTP at CAD\$50,000/QALY gain, probability HRA is cost-

effective are:-

- base case : $58 \%$

- male 60 years : 9\%

- male 40 years : 92\% - most

cost-effective

- The results and uncertainty in base-case analyses suggest that in terms of cost-effectiveness, there is little difference between MoM HRA and THA.

- In terms of gender, MoM HRA was preferable in men and THA in women

- Age wise, MoM HRA was preferable in younger patients and THA in older patients

- Base-case ICER : €2402 per "revision-free" life year

- Cementless strategy dominant for patients up to 42-y-old (i.e., less costly and more beneficial compared with the hybrid solution)

- 43-yr-old onwards, it still remains more effective but with an additional cost : the resulting
In summary, results suggest that THA is a cost-effective treatment option, and in general should be offered without delay to functionally independent patients with severe OA.

Hip and knee replacement both improve HRQoL. The cost per QALY gained from knee replacement is twice that gained from hip replacement.

MoM-HRA could be clinically advantageous and cost-effective in younger men and women. Further research on the comparative effectiveness of MoM-HRA versus THA should include assessments of the quality of life and resource use in addition to the clinical outcomes associated with both procedures.

On average, MoM-HRA was preferred to THA for younger and male patients, but THA is still a reasonable option if the patient or clinician prefers given the small absolute differences between the options and the confidence ellipses around the costeffectiveness estimates.

Following a deterministic sensitivity analysis, hybrid and cementless fixation showed a dominance profile for patients older than $83 \mathrm{y}$ and younger than $43 y$, whereas for all ages in between, there is a progressive increase in the ICER of cementless prostheses. 
Table 2 Summary of main results (Continued)

16. Pennington et al, [41]

17. Konopka et al, [33]

18. Marsh et al, [19]
High tibial osteotomy (HTO) and unicompartmental knee arthroplasty (UKA)

Arthroscopic surgery (partial resection and debridement of degenerative meniscal tears and/ or articular cartilage) in addition to non-operative treatments

CERs showed a direct propor-

tionality to increasing age

- From CEAC:

- the cementless intervention as a strategy with a high probability (0.88) of being cost effective at 70 y from values of WTP above $€ 2400$

- In case of a 75-y-old patient with WTP of $€ 9000$, a cementless approach results cost effective with a probability of 0.89 .

Cementless and hybrid prosthesis for THA
- The ICER for a hybrid prosthesis compared with a cemented prosthesis was about $£ 2100$ per QALY for men and £2500 for women.

- For men aged 60 or 80 and for women aged 60, hybrid prostheses gave the highest expected net benefit and had the highest probability of being the most cost effective prosthesis.

- For women aged 80, cemented prostheses were most cost effective.

- Hybrid prostheses remained likely to be the most cost effective option for men and women aged 70.

- Base case QALYs : 14.62 (HTO), 14.63 (UKA) and 14.64 (TKA). - Discounted total direct medical costs : \$20,436 (HTO), \$24,637 (UKA) and $\$ 24,761$ (TKA)

- ICER for TKA: \$231,900/ QALY

- ICER for UKA: \$420,100/ QALY

- PSA: At a WTP threshold of $\$ 50,000$ per QALY, HTO was costeffective $57 \%$ of the time; TKA

24\%; and UKA 19\%.

- At a WTP threshold of $\$ 100,000$ per QALY, high HTO $43 \%$ of time, TKA 31\%; and UKA 26\%.

- The ICER was $\$ 140.94$ (societal), or $\$ 120.83$ (payer) per one-point improvement on the 2400 point WOMAC total score, translating to $\$ 28,188$ (societal) and $\$ 24,166$ (payer) for a clinically important improvement.

- The ICUR was equal to

- \$110,569 (societal) or

- \$94,792.50 (payer) per QALY

gained, where the negative value indicates paying more for a worse outcome.

- Uncertainty estimates suggest that even if WTP $\$ 400000$ to achieve a clinically important improvement in WOMAC score, or $\geq \$ 50000$ for an additional QALY, there is $<20 \%$ probability that the addition of arthroscopy is cost-effective compared with nonoperative therapies only.
- Cemented prostheses are the cheapest option, but hybrid prostheses lead to greater gains in mean post-operative quality of life and are the most cost effective alternative for most patients. - Cementless prostheses do not improve health outcomes sufficiently to justify their higher costs.

In 50 to 60-year-old patients with medial unicompartmental knee OA, HTO is an attractive option compared with UKA and TKA The cost-effectiveness of HTO and of UKA depends on rates of conversion to TKA and the clinical outcomes of the conversions.

Arthroscopic debridement of degenerative articular cartilage and resection of degenerative meniscal tears in addition to nonoperative treatments for knee $\mathrm{OA}$ is not an economically attractive treatment option compared with non-operative treatment only, regardless of willingness-to-pay value. 
Table 2 Summary of main results (Continued)

19. Mather et al, [20] Primary TKA without delay

20. Peersman et al, [34]

UKA

21. Pennington et al, [23]

Different brands within types of hips prosthesis (cemented, cementless and hybrid)

22. Pulikottil-Jacob et al, [40] - Metal head (cemented stem) on cemented polyethy-lene cup, CeMoP

- Metal head (cement-less stem) on cement-less hydroxyapetite coated metal cup (polyethylene liner), CeLMoP

- Ceramic head (cementless stem) on cementless hydroxylapetite coated metal cup (ceramic liner), CeLCoC - Hybrid metal head (cemented stem) on cementless hydroxylapetite coated metal cup (polyethylene liner), HyMoP
- In the base case, a 2-year waittime both with and without a non-operative treatment bridge resulted in a lower number of average QALYs gained (11.57 (no bridge) and 11.95 (bridge) vs. 12.14 (no delay).

- The ICER comparing wait-time with no bridge to TKA without delay was $\$ 2,901 /$ QALY.

- When comparing TKA without delay to waiting with nonoperative bridge, TKA without delay produced greater utility at a lower cost to society.

- UKA was associated with cost reduction compared with primary TKA of $-€ 2,807$ and a utility gain of 0.04 QALYs. UKA was therefore considered superior to TKA.

- Analysis determined that the model is sensitive to clinical effectiveness, and that a marginal reduction in the clinical performance of UKA would lead to TKA being the more costeffective solution.

- The acceptability curve shows that the probability that the ICER falls below the threshold of $€ 10,000(77.1 \%)$, $€ 25,000(65.1 \%)$ and $€ 50,000$ (60.5\%).

For women with $\mathrm{OA}$ aged 70 years, the Exeter V40 Elite Plus Ogee had the lowest risk of revision (5.9\% revision risk, 9.0 QALYS) and the CPT Trilogy had the highest QALYs (10.9\% revision risk, 9.3 QALYS). - Compared with the Corail Pinnacle (the most commonly used brand), the CPT Trilogy is most cost effective, with an incremental net monetary benefit of $£ 876$.

- Differences in cost effectiveness between the hybrid CPT Trilogy and Exeter V40 Trident and the cementless Corail Pinnacle and Taperloc Exceed were small.

- base-case analysis : At a WTP £20,000 per QALY, a cemented prosthesis with metalonpolyethylene or ceramic-onpolyethylene bearings had the greatest probability of being cost-effective for all groups of age and gender over a lifetime. - The differences in QALYS between categories were extremely small and differences in mean costs were borderline, between only $£ 2550$ and $£ 3000$ over a lifetime for all comparisons, irrespective of age or gender.
TKA without delay is the preferred cost-effective treatment strategy when compared to a waiting for TKA without nonoperative bridge. TKA without delay is cost saving when a nonoperative bridge is used during the waiting period. As it is unlikely that patients waiting for TKA would not receive nonoperative treatment, TKA without delay may be an overall costsaving health care delivery strategy.

UKA yields clear advantages in terms of costs and marginal advantages in terms of health effects, in comparison with TKA.

The hybrid CPT-Trilogy was the 57 most cost effective brand but differences with the hybrid Exeter V40-Trident and the cementless Corail-Pinnacle and TaperlocExceed were small. Our study shows the importance of linking PROMs with data on rates of revision after THA but given the extended period of recovery after a THA, collecting further PROMs and QoL beyond the first six months after THA is an important next step which would strengthen future economic evaluations of brands of hip prostheses.

On the basis of such smal differences and such considerable uncertainties, it is difficult to make a comparison between the cost-effectiveness of different types of prosthesis. Until better data dealing with costs and outcomes become available, it is difficult to justify the recommendation of one type of device over another on considerations of cost effectiveness alone. The choice of prosthesis should be determined by rates of revision, local costs and the preferences of both the surgeon and the patient 
Table 2 Summary of main results (Continued)

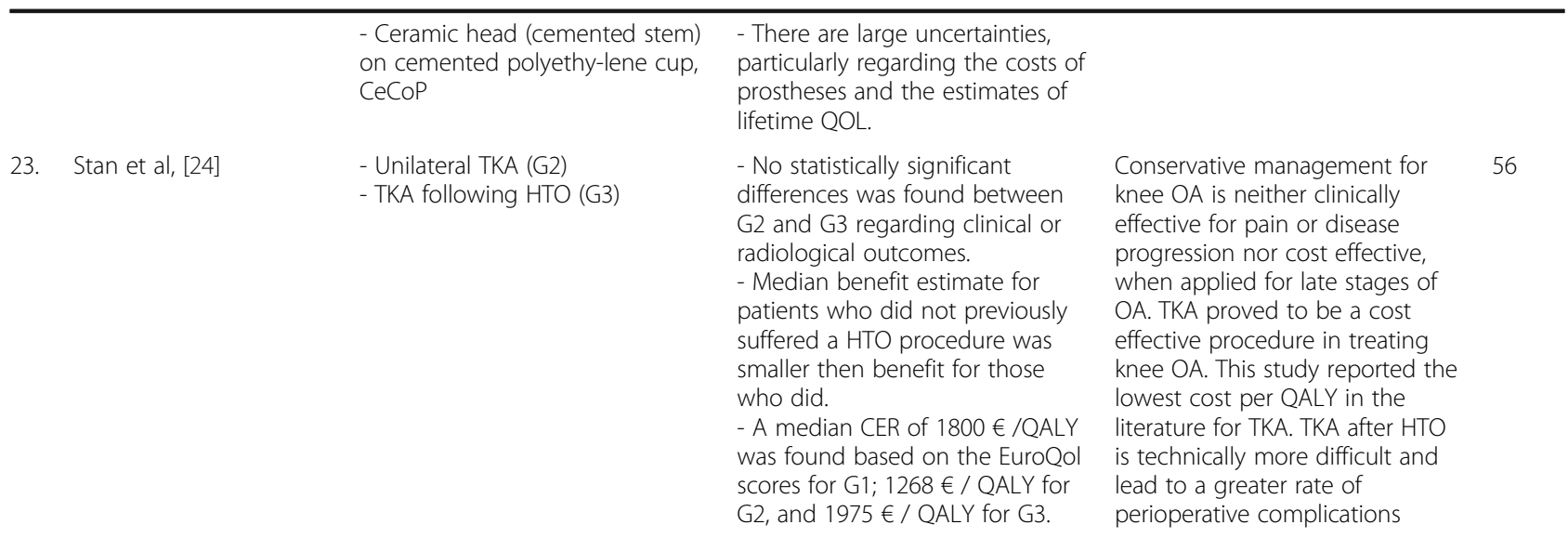

${ }^{\mathrm{a} G o o d}$ quality $=\geq 75$ Moderate quality $=50$ to 74 Poor quality $=<50$

UKA and THO and that HTO provided value for money in 50 to 60 year old patients. A summary of results are presented in Table 2 .

\section{TKA versus other treatments}

One study compared TKA with a do nothing approach over patients lifetime in the Australian population from a health system perspective [35], and found TKA to be a cost-effective intervention. Another study [20] compared early and delayed TKA (with and without a non operative bridge) in a cohort of 60 year olds from a societal perspective. The study found that TKA without delay is the preferred cost-effective treatment strategy when compared to waiting for TKA without non-operative bridge. This study noted that as it is unlikely that patients waiting for TKA would not receive non-operative treatment, TKA without delay may be an overall costsaving health care delivery strategy.

Another study [26] compared two types of TKA techniques (standard and innovative TKA) and concluded that innovative implants would be cost-effective if they reduce TKA failure by approximately 50\%. Finally, Losina et al [36] used a Markov model to simulate costs and QALY gains for TKA, carried out in low, medium and high volume hospitals; and using no TKA as comparator. Findings relate to the Medicare aged population in the US. TKA was found to be cost-effective across all risk groups and, usurpingly, to be more cost-effective when carried out in high volume hospitals (Table 2).

\section{Cost-effectiveness of Total Hip Arthroplasty (THA) THA vs Metal on metal hip resurfacing arthroplasty (MoM-HRA)}

Metal-on-metal hip resurfacing arthroplasty (MoMHRA) was considered as an alternative in two studies $[29,37]$. The first study used a Markov model to compare the cost-effectiveness of THA and MoM-HRA in patients aged 50 years and over from a health system perspective over a 30 year period and the results show that MoM-HRA is cost-effective in younger patients and although MoM-HRA showed improvements in QALYs, further research is needed to reach a definite conclusion as to which is the most cost-effective intervention [29]. The second study used a Markov model to assess the cost-effectiveness of THA compared with MoM-HRA from a health system perspective over a 15 year period. The study concluded that age wise, MoM-HRA was preferable in younger patients and THA in older patients and gender wise, MoM-HRA was more preferable in men and THA in women [37].

\section{THA vs other strategies}

One study compared THA to a non-operative strategy from a health care perspective and found THA to be a cost-effective intervention [28]. Another study compared the intervention to a do nothing approach over patients' lifetime in the Australian population from a health system perspective and found THA to be cost-effective [35]. The study by Mota used a Markov modelling approach to compare early and delayed hip arthroplasty in a cohort of patients based on sex and age (50-59 years, 60-74 years and 75 years and over) from a health provider perspective and the results from this study suggest that early THA is a cost-effective option across groups based on age and sex [38].

Four studies compared various techniques for THA. The first study [39] compared two techniques for THA (cementless and hybrid) over patients lifetime using a Markov model and found the cementless technique to be cost-effective compared to the hybrid technique in most cases. However, the cementless technique was found to be dominant in patients less than 43 years whilst the hybrid technique seemed to be dominant in patients aged 83 and over. 
The second study assessed the lifetime costeffectiveness of cemented, cementless and hybrid techniques and found the hybrid to be the most costeffective compared to cemented and cementless in 60, 70 and 80 year old patients. The results obtained in this study were similar for both men and women [23]. The third study [40] compared five different techniques for THA from an NHS/PSS perspective and concluded that it is difficult to make a choice between techniques based on cost-effectiveness grounds alone. The fourth study assessed the cost-effectiveness of three types of prosthesis for hip replacement (cemented, cementless and hybrid) in adults aged between 55 and 84 years. The study found the hybrid prosthesis to be the most costeffective across all age groups, with the exception of 80 year old women where the cemented prosthesis was the most cost-effective [41].

\section{Cost-effectiveness of other surgical interventions}

For studies that considered implant systems, one study evaluated the KineSpring implant system and found it to be cost-effective compared to other surgical and conservative treatments [22]. Another study compared arthroscopic surgery with a non-operative treatment and found that arthroscopic surgery was not a cost-effective option [19]. A summary of the results and conclusions of studies assessing the cost-effectiveness of surgical interventions is presented in Table 2 .

\section{Overall quality of included studies}

Based on the Quality of Health Economic Studies (QHES) instrument, a total of six studies were categorised as good quality with scores of 75 and above whilst fourteen other studies were categorised as moderate quality with scores between 50 to 74 (Table 2 and Additional file 1). Three studies had scores below 50 [21, 22, 28] and a close examination of these studies revealed striking flaws such as not reporting the source of transition probabilities, perspectives, discount rate, cycle length and time horizon. One study [22] did not perform any sensitivity analysis to deal with the sampling uncertainty or give any details of the source of effectiveness data.

Fourteen out of the eighteen model based studies used Markov/semi-Markov models due to the chronic nature of OA and the ability of such models to handle recurrent events. Other model types that were used include decision tree modelling [25] and discrete-event simulation (DES) which has the flexibility to accommodate a richer structure without making it unmanageable in size [35]. Only one study did not specify which model type was used as the basis of its economic evaluation [22]. The time horizon used in the model based studies varied from one year to lifetime and most of the studies chose a 1-year cycle length for transitions to occur from one health state to another. For those studies that accounted for revision of surgery and failure of implant as one of the outcomes, one year is considered as a reasonable time to decide for further management, should any of the events occur. The most commonly used sensitivity analysis in the model-based economic evaluations in this review is probabilistic sensitivity analysis. This involves specifying distributions for model parameters to represent uncertainty in their estimation followed by employing Monte Carlo simulation to select values at random from those distributions [42]. Other types of sensitivity analysis used were deterministic, one-way and two-way sensitivity analysis, subgroup analysis, regression analysis and scenario analysis.

\section{Discussion}

This systematic review was conducted with the objective of assessing the cost-effectiveness of surgical interventions for the treatment of knee and hip OA. Overall, the review found that most studies considered the most important components pertinent to economic analyses such as perspective, currency, price year and time horizon. Total knee arthroplasty, total hip arthroplasty and metal-on-metal hip resurfacing arthroplasty showed evidence of cost-effectiveness and improvement in quality of life of the patients. However, this depended on the population that was considered and the interventions that they were compared to. For example, TKA and THA were found to be cost-effective for patients with severe or end-stage knee or hip OA across all age groups and when compared to non-operative strategies. Regardless of perspective adopted, TKA and THA remain among the most effective interventions in terms of improvements in quality of life of OA patients. Compared to early primary THA or TKA, the findings suggest that delayed total hip arthroplasty was not cost-effective [38]. MoM-HRA surgery was more cost-effective in younger patients.

More than half of the studies in this review used decision-analytic models and as expected, some of the earlier studies $[25,43]$ used decision trees to analyse the cost-effectiveness of the interventions of interest, whilst the more recent studies utilised Markov models. This is an indication that adequate information on model structures is now readily available and guidelines are being adhered to. Based on the chronic nature of the disease and potential of recurrence of the events that may occur during disease progression in OA, Markov models have been shown to be the most suitable decision-analytic model for this condition since they provide a far more convenient way of modelling prognosis for clinical problems with ongoing risk whereby events may re-occur and when the utility of an outcome depends on when it occurs [44]. One study employed a discrete-event 
simulation (DES) model to assess the cost-effectiveness of total hip and knee arthroplasty in Australia [35]. The literature has shown that DES is able to represent the course of a disease more naturally and is best used in a condition where interaction at individual or patient level is a significant component in modelling [45, 46].

\section{Strengths and limitations of the study}

To the best of our knowledge, this is the only systematic review pertaining to the cost-effectiveness of surgical treatment modalities in management of osteoarthritis. In addition, methodological issues related with economic evaluations of surgical interventions in management of OA were also assessed and reported in this study. This study critically assessed the quality of included studies both qualitatively with the Philips and CHEERS checklists as well as quantitatively using the QHES scoring tool.

Because a broader search strategy was initially adopted, a large number of studies were hand searched. This may mean that we have been able to identify a larger number of relevant papers than if the initial search criteria had been limited to surgical interventions for hip and knee OA.

Limitations of the study include the following: Due to the study aims and the need to include as many studies as possible, we did not exclude any studies based on the results of the quality checks. Hence, the robustness of this systematic review may possibly be affected by the poor quality studies included in this report. In addition to this, the quality assessment was carried out by one reviewer. Second, studies that were included in this review were conducted across nine countries which may lead to problems such as generalisability and transferability of the study findings to other settings due to differences in factors such as clinical practice, prices and epidemiology of disease $[47,48]$. In addition to this, guidance in many countries does not consider economic data to be transferable to their settings in most cases $[49,50]$. As a result, care must be taken when interpreting the results of this study in a particular context.

\section{Policy implications and recommendations}

Clinical guidelines by the National Institute for Health and Care Excellence in the UK recommend a holistic approach to osteoarthritis assessment and management [8]. The guidelines advocate for patient education and self-management to enhance understanding of the condition and its management. Unfortunately, the information on economic analyses in the guidelines is confined to pharmacological and conventional treatments. Costeffectiveness findings related to surgical interventions were merely focused on time to referral for surgery as being highlighted in the guidelines by National
Collaborating Centre for Chronic Conditions [51]. It is therefore recommended that guidelines should also include suggestions for the use of surgical interventions as well as other forms of treatments and management techniques. One of the concerns around surgical interventions such as arthroplasty relates to the cost implications surrounding it. It may be argued that avoiding or delaying these surgical procedures may have a positive impact on health budgets through savings. However, it should be noted that delaying such a procedure may have a detrimental effect on the quality of life of patients and may lead to additional costs down the line. Studies included in this review concluded that delaying arthroplasty (both knee and hip) was not a cost-effective option and that the health related quality of life lost as a result of the delay is greater than the savings in costs that may occur from delaying the procedure [38]. It is therefore suggested that future cost-effectiveness studies assess the cost-effectiveness of delaying surgery in various subgroups of patients and in various settings in order to reach a consensus about when the operation should be conducted and when delaying surgery would yield the optimum results.

Although interventions such as THA have been shown to be cost-effective, there needs to be additional studies comparing the various types of THA surgeries in order to maximise benefits from the available resources. In addition, other interventions such as MoM-HRA have been shown to be cost-effective in younger patients [37]. Therefore health policy makers should take such evidence into consideration when providing guidance. Policy makers and stakeholders should therefore consider TKA and THA as opposed to non-operative/non surgical strategies particularly for patients with severe/advanced stage OA [18]. From this review, it is clear that there is limited evidence with respect to other forms of surgical treatments. It is therefore suggested that additional studies should be conducted in order to determine the cost-effectiveness of other forms of surgical interventions for both knee and hip OA.

This study found that approximately $36 \%$ percent of studies limited their analysis to a healthcare perspective. It should however be noted that a large proportion of the economic burden of OA is related to indirect costs and productivity losses [6]. Thus, the societal perspective has the potential to capture all important impacts on the whole society. Recent guidelines suggest that due to the chronic nature of $\mathrm{OA}$, a broader societal perspective is preferred $[9,52]$. It should however be recognised that national guidelines such as those in the UK recommend that economic analysis should be undertaken from a health service perspective [53] and as a result, analysts in countries like the UK might limit their study to the health service perspective. However, it is suggested that 
all studies should also consider the societal perspective within sensitivity analysis in order to provide a better picture of the true burden associated with $\mathrm{OA}$ and also ensure that the results are more generalisable to other settings.

\section{Conclusions}

This review assessed the cost-effectiveness of surgical interventions for the management of knee and hip osteoarthritis and the results suggest that TKA and THA are cost-effective interventions particularly when compared to non-operative strategies and also when the operation is not delayed. However, there is the need for more studies assessing the cost-effectiveness of other surgical modalities. In addition, most of the identified studies were considered to have moderate quality. It is therefore recommended that more cost-effectiveness studies with high methodological standards are conducted.

\section{Additional file}

Additional file 1: Search strategy for electronic databases. (DOCX $33 \mathrm{~kb}$ )

\section{Abbreviations}

CHEERS: Consolidated health economic evaluating reporting standard; DES: Discrete event simulation; EQ-5D: EuroQoL five dimension; HAQ: Health assessment questionnaire; HR-QoL: Health related quality of life; HTO: High tibial osteotomy; HUI: Health utility index; ICER: Incremental costeffectiveness ratio; MoM-HRA: Metal on metal hip resurfacing arthroplasty; OA: Osteoarthritis; QALY: Quality adjusted life years; QHES: Quality of health economics studies; SF-36: Short form 36; SF-6D: Short form 6D; THA: Total hip arthroplasty; TKA: Total knee arthroplasty; UKA: Unicondylar knee arthroplasty

\section{Funding}

Not Applicable.

\section{Availability of data and materials}

All data has been summarised in the results section and the tables.

\section{Authors' contributions}

All authors contributed extensively to the work presented in this paper. HK $\mathrm{PK}$ and $\mathrm{RO}$ developed the research question. HK and RO conducted the searches. HK, PK and RO interpreted the data. All authors contributed to drafting the paper and provided comments throughout. All authors approved the final version of the paper.

\section{Competing interests}

The authors declare that they have no competing interest.

\section{Consent for publication}

Not Applicable.

\section{Ethics approval and consent to participate}

Not applicable.

\section{Publisher's Note}

Springer Nature remains neutral with regard to jurisdictional claims in published maps and institutional affiliations.

\section{Author details}

'Malaysian Health Technology Assessment Section, Ministry of Health, Putrajaya, Malaysia. ${ }^{2}$ Health Economics Unit, Institute of Applied Health Research, University of Birmingham, Birmingham, UK.

Received: 23 June 2016 Accepted: 28 April 2017

Published online: 10 May 2017

\section{References}

1. Wang TJ, et al. Effects of aquatic exercise on flexibility, strength and aerobic fitness in adults with osteoarthritis of the hip or knee. J Adv Nurs. 2007; 57(2):141-52.

2. Cross $M$, et al., The global burden of hip and knee osteoarthritis: estimates from the Global Burden of Disease 2010 study. Annals of the rheumatic diseases, 2014. doi:10.1136/annrheumdis-2013-204763

3. Office of National Statistics. Statistical Bulletin: Estimates of the very old (including centenarians) for the United Kingdom, 2002-2012. 2014 [cited 2016; Available from: www.ons.gov.uk/ons/dcp171778_357100.pdf. Accessed 4 May 2017.

4. United Nations Department of Economic and Social Affairs. World Population Ageing 2013. 2013 [cited 2016; Available from: http://www.un. org/en/development/desa/population/publications/pdf/ageing/ WorldPopulationAgeing2013.pdf. Accessed 4 May 2017.

5. Le TK, et al. Health care costs in US patients with and without a diagnosis of osteoarthritis. J Pain Res. 2012;5:23-30.

6. Chen $\mathrm{A}$, et al. The global economic cost of osteoarthritis: how the UK compares. Arthritis. 2012;2012:698709.

7. Kotlarz $\mathrm{H}$, et al. Insurer and out-of-pocket costs of osteoarthritis in the US: evidence from national survey data. Arthritis Rheumatol. 2009;60(12): 3546-53.

8. NICE. Osteoarthritis: care and management in adults 2014. 2014 [cited 2016; Available from: https://www.nice.org.uk/guidance/cg177. Accessed 4 May 2017

9. Hilismann, M., et al., Health economics in the field of osteoarthritis: an expert's consensus paper from the European Society for Clinical and Economic Aspects of Osteoporosis and Osteoarthritis (ESCEO). Seminars in arthritis and rheumatism, 2013: p. 303-313.

10. Kurtz SM, et al. Future young patient demand for primary and revision joint replacement: national projections from 2010 to 2030. Clin Orthop Relat Res. 2009;467:2606-12.

11. Piscitelli $P$, et al. Socioeconomic burden of total joint arthroplasty for symptomatic hip and knee osteoarthritis in the Italian population: a 5-year analysis based on hospitalization records. Arthritis Care Res. 2012;64:1320-7.

12. Pinto $D$, et al. Cost-effectiveness of nonpharmacologic, nonsurgical interventions for hip and/or knee osteoarthritis: systematic review. Value Health. 2012;15(1):1-12.

13. Wielage RC, et al. Cost-effectiveness analyses of osteoarthritis oral therapies: a systematic review. Appl Health Econ Health Policy. 2013:11(6):593-618.

14. Xie F, et al. Cost-effectiveness of pharmaceutical management for osteoarthritis pain. Drugs Aging. 2013:30(5):277-84.

15. Husereau D, et al. Consolidated health economic evaluation reporting standards (CHEERS) — explanation and elaboration: a report of the ISPOR health economic evaluation publication guidelines good reporting practices task force. Value Health. 2013;16(2):231-50.

16. Philips $Z$, et al. Review of guidelines for good practice in decision-analytic modelling in health technology assessment. Health Technol Assessment. 2004:8:36.

17. Ofman JJ, et al. Examining the value and quality of health economic analyses: implications of utilizing the QHES. J Manag Care Pharm. 2003:9(1):53-61.

18. Bedair H, Cha TD, Hansen VJ. Economic benefit to society at large of total knee arthroplasty in younger patients. J Bone Joint Surg Am. 2014;96(2): 119-26.

19. Marsh JD, et al. Cost-effectiveness analysis of arthroscopic surgery compared with non-operative management for osteoarthritis of the knee. BMJ open. 2016;6(1):e009949.

20. Mather RC, et al. Economic evaluation of access to musculoskeletal care: the case of waiting for total knee arthroplasty. BMC Musculoskelet Disord. 2014;15(1):1.

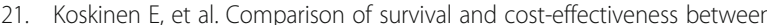
unicondylar arthroplasty and total knee arthroplasty in patients with primary osteoarthritis: a follow-up study of 50,493 knee replacements from the Finnish Arthroplasty Register. Acta Orthopaedica. 2008;79(4):499-507. 
22. Li CS, et al. Cost-effectiveness and economic impact of the KineSpring ${ }^{\oplus}$ Knee Implant System in the treatment for knee osteoarthritis. Knee Surg Sports Traumatol Arthrosc. 2013;21(11):2629-37.

23. Pennington $M$, Grieve $R$, van der Meulen JH. Lifetime cost effectiveness of different brands of prosthesis used for total hip arthroplasty a study using the NJR dataset. Bone Joint J. 2015;97(6):762-70.

24. Stan G, Orban H, Orban C. Cost effectiveness analysis of knee osteoarthritis treatment. Chirurgia. 2015;110:368-74.

25. Soohoo NF, et al. Cost-effectiveness analysis of unicompartmental knee arthroplasty as an alternative to total knee arthroplasty for unicompartmental osteoarthritis. J Bone Joint Surg. 2006;88(9):1975-82.

26. Suter $L G$, et al. Placing a price on medical device innovation: the example of total knee arthroplasty. PloS One. 2013;8(5):e62709.

27. Xie F, et al. Total or partial knee replacement? Cost-utility analysis in patients with knee osteoarthritis based on a 2-year observational study. Eur J Health Econ. 2010;11(1):27-34

28. Räsänen $\mathrm{P}$, et al. Effectiveness of hip or knee replacement surgery in terms of quality-adjusted life years and costs. Acta Orthop. 2007;78(1):108-15.

29. Bozic KJ, et al. Do the potential benefits of metal-on-metal hip resurfacing justify the increased cost and risk of complications? Clin Orthop Relat Res. 2010:468(9):2301-12

30. Bellamy N, et al. Validation study of a computerized version of the Western Ontario and McMaster Universities VA3. 0 Osteoarthritis Index. J Rheumatol. 1997;24(12):2413-5.

31. Ruiz D, et al. The direct and indirect costs to society of treatment for endstage knee osteoarthritis. J Bone Joint Surg Am. 2013;95(16):1473-80.

32. Waimann CA, et al. Cost-effectiveness of total knee replacement: a prospective cohort study. Arthritis Care Res. 2014;66(4):592-9.

33. Konopka JF, et al. The cost-effectiveness of surgical treatment of medial unicompartmental knee osteoarthritis in younger patients. J Bone Joint Surg Am. 2015;97(10):807-17.

34. Peersman $\mathrm{G}$, et al. Cost-effectiveness of unicondylar versus total knee arthroplasty: a Markov model analysis. Knee. 2014;21:S37-42.

35. Higashi $\mathrm{H}$, Barendregt JJ. Cost-effectiveness of total hip and knee replacements for the Australian population with osteoarthritis: discreteevent simulation model. PLoS One. 2011;6(9):e25403.

36. Losina $E$, et al. Cost-effectiveness of total knee arthroplasty in the United States: patient risk and hospital volume. Arch Intern Med. 2009;169(12):1113.

37. Heintzbergen $\mathrm{S}$, et al. Cost-utility of metal-on-metal hip resurfacing compared to conventional total hip replacement in young active patients with osteoarthritis. Value Health. 2013;16(6):942-52.

38. Mota R. Cost-effectiveness analysis of early versus late total hip replacement in Italy. Value Health. 2013;16(2):267-79.

39. Di Tanna GL, et al. Modeling the cost-effectiveness for cement-less and hybrid prosthesis in total hip replacement in Emilia Romagna, Italy. J Surg Res. 2011;169(2):227-33.

40. Pulikottil-Jacob R, et al. Cost effectiveness of total hip arthroplasty in osteoarthritis comparison of devices with differing bearing surfaces and modes of fixation. Bone Joint J. 2015;97(4):449-57.

41. Pennington $M$, et al., Cemented, cementless, and hybrid prostheses for total hip replacement: cost effectiveness analysis. BMJ, 2013. 346 doi:10.1136/bmj. f1026

42. Griffin S, Claxton K. Analyzing uncertainty in cost-effectiveness for decisionmaking. In: Glied S, Smith PC, editors. The Oxford Handbook of Health Economics. New York: Oxford University Press; 2011. p. 759-87.

43. Soohoo N, Kominski G. Cost-effectiveness analysis of total ankle arthroplasty. J Bone Joint Surg Am. 2004;86(11):2446-55.

44. Sonnenberg FA, Beck JR. Markov models in medical decision making a practical guide. Med Decis Mak. 1993;13(4):322-38.

45. Barton P, Bryan S, Robinson S. Modelling in the economic evaluation of health care: selecting the appropriate approach. J Health Serv Res Policy. 2004:9(2):110-8

46. Caro J. Pharmacoeconomic analyses using discrete event simulation. PharmacoEconomics. 2005:23(4):323-32.

47. Hughes D, et al. Conducting economic evaluations alongside randomised trials: current methodological issues and novel approaches. PharmacoEconomics. 2016;34(5):447-61.

48. Koopmanschap MA, Touw KC, Rutten FF. Analysis of costs and costeffectiveness in multinational trials. Health Policy. 2001;58(2):175-86.

49. Barbieri $\mathrm{M}$, et al. What do international pharmacoeconomic guidelines say about economic data transferability? Value Health. 2010;13(8):1028-37.
50. Oppong R, Jowett S, Roberts TE. Economic evaluation alongside multinational studies: a systematic review of empirical studies. PloS One. 2015;10(6):e0131949.

51. NICE. Osteoarthritis: national clinical guideline for care and management in adults. 2008 [cited 2015; Available from: https://www.nice.org.uk/guidance/ cg59. Accessed 4 May 2017.

52. Hiligsmann $M$, et al. A reference case for economic evaluations in osteoarthritis: An expert consensus article from the European Society for Clinical and Economic Aspects of Osteoporosis and Osteoarthritis (ESCEO). Semin Arthritis Rheum. 2014;44:271-82.

53. NICE, Guide to the Methods of Technology Appraisal 2013. Process and Methods Guides, 2013. 04 April https://www.nice.org.uk/process/pmg9/ resources/guide-to-the-methods-of-technology-appraisal-2013-pdf2007975843781. Accessed 4 May 2017.

\section{Submit your next manuscript to BioMed Central and we will help you at every step:}

- We accept pre-submission inquiries

- Our selector tool helps you to find the most relevant journal

- We provide round the clock customer support

- Convenient online submission

- Thorough peer review

- Inclusion in PubMed and all major indexing services

- Maximum visibility for your research

Submit your manuscript at www.biomedcentral.com/submit
C) Biomed Central 